\title{
Aspectos críticos de la concepción de Piaget sobre los números
}

\author{
José I. Navarro*
}

\begin{abstract}
Resumen
De acuerdo con las teorías constructivistas de Piaget, para que los niños pequeños aprendan matemáticas, es necesario enseñarles primero los procesos lógicos y su organización, puesto que estos serían "prerrequisitos" para la adquisición del concepto de número. De acuerdo con la reciente investigación científica contrastada sobre aprendizaje de las matemáticas, esta concepción piagetiana de la adquisición del número en el niño no está suficientemente justificada. Es claro que los escolares tienen mucho que aprender sobre aritmética y su comprensión del número mejora con la edad y con el aprendizaje, pero eso no significa que carezcan de nociones numéricas acertadas antes de llegar a la educación infantil, incluso desde el nacimiento. Piaget diseñó ingeniosos procedimientos para estudiar la adquisición de los prerrequisitos del número (seriación, conservación, correspondencia), pero lo que nos dice la investigación empírica al respecto es que cuando a los niños de 3 a 6 años se les presentan situaciones análogas a las ideadas por Piaget, de características no verbales, sus habilidades con los números progresan vertiginosamente. En este trabajo, a partir del experimento clásico de Piaget sobre la conservación del número, se analiza la teoría piagetiana de manera crítica desde los resultados encontrados en experimentos paralelos.
\end{abstract}

Palabras clave: conservación del número, contexto, errores, motivación, tareas de conservación.

\section{Critical Aspects of Piaget's Conception of Numbers}

\begin{abstract}
According to the constructivist theories of Piaget, in order for small children to learn mathematics, they must first be taught the logical processes and their organization, as these are prerequisites for acquiring number concepts. As shown in recent scientific research into mathematical learning, however, this Piagetian concept of number acquisition in children is not sufficiently justified. It is clear that children starting school have much to learn about arithmetic, and their understanding of numbers improves with age and learning, but this does not mean that they lack accurate notions of numbers before beginning their education, or even from birth. Piaget designed ingenious procedures to study the acquisition of the prerequisites for numeracy (seriation, conservation, correspondence), but empirical research tells us that when children aged from 3 to 6 are presented with non-verbal situations analogous to those designed by Piaget, their ability with numbers develops dramatically. This study looks at Piaget's classic experiment of number conservation and critically analyzes Piagetian theory based on the results of parallel experiments.
\end{abstract}

Keywords: number conservation, context, errors, motivation, conservation tasks

\section{Aspectos críticos da concepção de Piaget sobre os números}

\section{Resumo}

De acordo com as teorias construtivistas de Piaget, para que as crianças aprendam matemática, é necessário ensinar-lhes primeiro os processos lógicos e sua organização, já que estes seriam "pré-requisitos" para a aquisição do conceito de número. Segundo a recente pesquisa científica contrastada sobre aprendizagem da matemática, essa concepção piagetiana da aquisição do número na criança não está suficientemente justificada. É claro que as crianças em idade escolar têm muito que aprender sobre aritmética e sua compreensão do número melhora com a idade e com a aprendizagem, mas isso não significa que careçam de noções numéricas acertadas antes de chegar à educação infantil, inclusive desde o nascimento. Piaget desenhou engenhosos procedimentos para estudar a aquisição dos pré-requisitos do número (seriação, conservação, correspondência), mas a pesquisa empírica a respeito disso indica que, quando situações análogas às idealizadas por Piaget, de características não verbais, são apresentadas às crianças de três a seis anos, suas habilidades com os números progridem significativamente. Neste trabalho, a partir da experiência clássica de Piaget sobre a conservação do número, analisa-se a teoria piagetiana de maneira crítica a partir dos resultados encontrados em experiências paralelas.

Palavras-chave: conservação do número, contexto, erro, motivação, tarefas de conservação.
Cómo citar este artículo: Navarro J. I. (2014). Aspectos críticos de la concepción de Piaget sobre los números. Pensando Psicología, 10(17), 97-101. doi: http://dx.doi. org/10.16925/pe.v10i17.788 


\section{Introducción ${ }^{1}$}

En la mayoría de los centros de educación infantil de nuestro entorno, los escolares invierten una buena cantidad de tiempo poniendo en orden cubos de diferentes tamaños y cosas por el estilo antes de que se les enseñe a contar. De acuerdo con las teorías constructivistas de Piaget, es necesario enseñarles primero los procesos lógicos y su organización, puesto que estos serían "prerrequisitos" para la adquisición del concepto de número. Así se lo enseñamos en la Universidad a los futuros maestros y, en algunos casos, así lo ponen en práctica después. En realidad, consideramos que esta concepción piagetiana de la adquisición del número en el niño puede responder a una metodología basada en el análisis exhaustivo de pocos casos. Utilizando otro tipo de procedimientos, se ha encontrado experimentalmente que ratas y palomas reconocen con cierta facilidad números de objetos, incluso aunque cambiemos su configuración espacial (Piazza, Dehaene y Gazzaniga, 2004), o bien que los chimpancés eligen espontáneamente la mayor entre dos cantidades (Brannon, Cantlon y Terrace, 2006). Salvando todas las distancias metodológicas y filogenéticas, sería razonable pensar que los niños y niñas de la especie humana puedan tener la habilidad del conteo antes de los 4 o 5 años.

Hoy sabemos que Piaget no estaba acertado en este asunto del concepto de número. Es claro que los escolares tienen mucho que aprender sobre aritmética y que su comprensión del número mejora con la edad y con el aprendizaje, pero eso no significa que carezcan de nociones numéricas acertadas antes de llegar a la educación infantil, incluso desde el nacimiento, como han demostrado algunos investigadores (Karmiloff-Smith, 1996). Lo que se debe hacer para demostrarlo es poner en juego procedimientos experimentales a la medida de su edad. Infortunadamente, los test que usaba Piaget tenían un formato que no reflejaba lo que realmente sabían los niños en relación con los números. Su principal defecto era precisamente lo que Piaget consideraba era la clave de su método: el diálogo establecido entre el experimentador y los jóvenes participantes. El tema está en conocer si los niños podían saber lo que "realmente" se les estaba preguntando y, lo que es más importante, si son capaces de interpretar las preguntas en el sentido que pueden hacer los adultos (Dehaene,
1997). Existen varias y profundas razones para pensar que no. En realidad, lo que nos dice la investigación empírica al respecto es que cuando a los niños de 3 a 6 años se les presentan situaciones análogas a las que son sometidos los animales de laboratorio, y cuando su capacidad mental se prueba con test no verbales, sus habilidades con los números progresan vertiginosamente.

\section{Conservación de cantidades discretas}

Uno los test más característicos desarrollados por Piaget fue el de la conservación del número (Gréco, Inhelder, Matalon y Piaget, 1963). Mehler y Bever (1967) publicaron un artículo en Science poco divulgado entre los constructivistas. Estos psicólogos del prestigioso Massachusetts Institute of Technology demostraron que los resultados en la aplicación del test de conservación del número diseñado por Piaget cambiaban considerablemente según fuera el contexto y el nivel de motivación que tuvieran los niños de sus experimentos. Estudiando a niños y niñas de 2 a 4 años, les mostraban dos tipos de tareas. La primera era parecida al preparado experimental tradicional de Piaget: dos filas de canicas. La fila más corta tenía 6 canicas, y la más larga tenía 4 (ver figura 1). Cuando a los participantes se les pedía que señalaran la fila con más canicas, la mayoría de los que tenían 3 y 4 años se equivocaban, y señalaban la fila más larga, pero con menos canicas. Sin duda, esto hace pensar en un característico error de conservación según la explicación de Piaget. Ahora bien, a los citados autores se les ocurrió cambiar las canicas por sabrosos caramelos de chocolate (los famosos M\&M's, tan populares entre los niños norteamericanos), y en lugar de formularles preguntas complejas, simplemente los invitaban a que se comieran la fila que tenía más caramelos. Los resultados fueron diametralmente opuestos: la mayoría de los niños acertaban en elegir la fila con más M\&M's. Este procedimiento tenía una doble ventaja: por un lado, no utilizaba un lenguaje de dudosa comprensión para el niño, y por otro, la motivación por elegir la fila con más caramelos se veía favorecida. No obstante, a cualquier padre o madre no le sorprendería que sus hijos de 3 y 4 años eligieran la fila con más caramelos, aunque esto contradiga la teoría de Piaget.

Este trabajo fue financiado parcialmente con el proyecto edu201122747 del Plan Nacional I+D+I y P09-HUM7918 del Plan Andaluz de Investigación, Desarrollo e Innovación (PAIDI). 


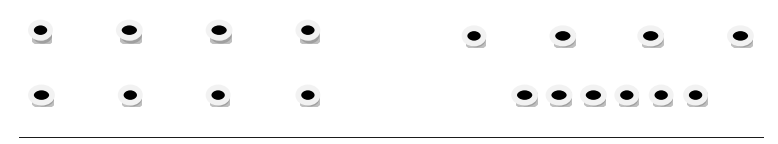

Figura 1. Formato de la tarea de conservación del número antes y después de la transformación

En el experimento de Mehler y Bever, se daba otra circunstancia sorprendente: los niños de 2 años acertaban sin error en la elección de la fila correcta, fueran canicas o fueran caramelos. Eran los niños y niñas mayores quienes cometían errores en el experimento con las canicas (no con los m\&m's). Es decir, parecería que la conservación del número aparecía muy pronto, para luego desaparecer en el curso evolutivo por arte de magia. Sin embargo, lo que conocemos sobre desarrollo cognitivo (Santrock, 2010) -y es una obviedad para cualquier padre o madre-, es que los niños mayores tienen mayores dominios mentales que los pequeños. $\mathrm{O}$ lo que es lo mismo: los preparados experimentales tal como fueron diseñados por Piaget simplemente no pueden evaluar adecuadamente el conocimiento numérico a esas edades. Como señala Dehaene (1997), tal como se formulan las preguntas parece que confunden a los niños mayores y, por eso, no responden tan bien como sus compañeros más pequeños.

\section{Lectura de las intenciones del hablante}

Otra interpretación más ajustada al conocimiento actual de la psicología cognitiva infantil podría ser la dada por autores como Thomas y Karmiloff-Smith (2003). Los niños de 3 y 4 años "interpretan" las preguntas de los experimentadores de una forma bien distinta de como a ellos les gustaría. Los términos utilizados y el contexto en el que se presentan inclinan a los niños a pensar que se les está preguntando por la longitud de las filas, no por la cantidad de elementos que hay en ellas. Recuerden que en el experimento original de Piaget se pregunta a los niños dos veces "¿Son iguales, 0 una fila tiene más canicas que la otra?”; la primera vez cuando la correspondencia es uno a uno, y la segunda cuando se ha cambiado la longitud de la fila.

Supongamos que es cierto que la igualdad numérica de las dos filas es inicialmente obvia para el niño. ¿Qué podrá pensar él de estas preguntas? Quizás piense que es raro que un señor ya bien crecidito le pregunte dos veces algo trivial. Verdaderamente es una violación de las elementales reglas de conservación hacer una pregunta como esa, más aún cuando ya ha contestado una vez que son iguales. Enfrentado con este conflicto interno, quizás los niños entiendan que la segunda pregunta -aunque superficialmente idéntica a la primera- no quiera decir lo mismo. Quizás el razonamiento de un niño de 4 años pueda ser algo parecido a lo siguiente:

Si este tipo tan mayor me pregunta dos veces lo mismo, será porque espera una respuesta diferente. Lo único que ha cambiado respecto a la situación anterior es la longitud de una de las filas. Por lo tanto, esta vez la pregunta debe referirse a la longitud, aunque me haya dicho que es sobre la cantidad. Así que le contestaré sobre la base de la longitud, más que teniendo en cuenta el número (Dehaene, 1997, p. 46).

Podemos entender que este razonamiento sería demasiado sofisticado para un niño de 4 años, pero su esencia entra dentro de las posibilidades intelectuales de la edad. Las inferencias de este tipo sobre las intenciones del hablante están muy a su alcance. Todos las hacemos de forma rutinaria; los niños también. La comprensión de una frase consiste en ir más allá de su sentido literal. En muchas circunstancias, el significado literal y el real pueden ser opuestos. Cuando nos preguntan si nos ha gustado una buena comida podemos contestar: "Pues no ha estado nada mal". O a veces esperamos ir más allá del sentido literal de una frase. Por ejemplo, si en el autobús ofrecemos nuestro asiento a una señora mayor, preguntándole “¿Quiere usted sentarse?”, esperamos que su respuesta sea algo más que un "si”" (más bien la respuesta esperada es: "Sí, gracias", o "sí, es usted muy amable"). Es decir, con frecuencia reinterpretamos las frases realizando complejas inferencias más allá del lenguaje literal, e interpretando las intenciones del hablante. Los niños a los 4 años pueden estar haciendo lo mismo cuando conversan con los adultos que les están administrando estos tipos de test. Es más, los que tenemos experiencia en la evaluación psicológica infantil, podemos constatar este hecho. Esta explicación puede ser la hipótesis más acertada ya que hacia los 4 años los niños comienzan a desarrollar la capacidad de razonar sobre las intenciones o las creencias de los demás, lo que los psicólogos llaman "teoría de la mente" (Frith, 2004).

\section{Un osito de peluche escocés}

Algunos experimentos han tratado de conocer en qué medida este juicio de intenciones del hablante puede estar vinculado con la conservación del número en los test de Piaget. En efecto, en la Universidad de Edim- 
burgo, McGarrigle y Donalson (1974) probaron si era la falta de comprensión de las intenciones del experimentador la responsable de las respuestas erróneas en estas tareas de conservación. Diseñaron un experimento sobre la conservación del número con 80 niños de 4 a 6 años, en el que la mitad de los ensayos eran clásicos: el experimentador alteraba la longitud de la fila y le preguntaba al niño cuál tenía más. Pero la otra mitad de los ensayos se hacía de manera bien diferente. Los cambios en la longitud de la fila los hacía un oso de peluche de manera disimulada, haciendo como que el experimentador no se había dado cuenta de lo que el osito había hecho. Mientras el experimentador miraba al tendido, el osito alargaba una de las filas de canicas. De pronto, aquel se daba cuenta del cambio y decía: “¡Oh no, este osito me ha desordenado todo! ¿Cuál tenía más?". La mayoría de los niños respondían correctamente en esta situación. La hipótesis era que así no habría dudas de que el niño conocería sin ambigüedad cuál era la intención del experimentador con su pregunta de "cuál tenía más": la petición era sincera y podría ser interpretada en el sentido literal. Sin embargo, estos mismos niños respondían erróneamente cuando era el experimentador quien cambiaba la longitud de las filas. Los autores consiguieron resultados similares cuando se evaluaba la conservación de cantidades continuas (Donaldson y McGarrigle, 1974, p. 189). Este sencillo experimento nos permite señalar dos cosas: un niño a esta edad ya es capaz de interpretar la misma pregunta de dos formas distintas según el contexto en el que se presenten. Cuando a un niño de esta edad se le hace una pregunta en un contexto coherente, responde correctamente porque es capaz de conservar el número.

En fin, el lector atento no debe sacar la conclusión de que las aportaciones de Piaget carecen de importancia. Leer a Piaget sigue siendo fundamental. Hemos tenido la suerte de poder hablar con dos alumnos directos suyos: los doctores Juan Pascual-Leone y Marc Richelle. Ambos coinciden en que era un genio irrepetible.

Tampoco debemos sacar la conclusión de que los fallos de los niños en las tareas de conservación son cuestiones triviales. Por el contrario, la adquisición del número en los escolares nos atrae mucho y es materia de estudio para un amplio número de investigadores (Navarro et al., 2012; Swanson, Jerman y Zheng, 2008). Después de numerosos experimentos, todavía no sabemos exactamente por qué los niños se dejan engañar tan fácilmente en las tareas de conservación a partir de estímulos falaces, tales como la longitud de las filas.
Ciertos neurocientíficos consideran que estos fallos en las tareas piagetianas tienen mucho que ver con el proceso madurativo del cortex prefrontal, una zona del cerebro que nos capacita para decidir una estrategia y mantenernos firmes en ella, a pesar de las distracciones (Ouden, Frith, Frith y Blakemore, 2005). Si esto es así, las pruebas de conservación de Piaget podrían ser una buena manera de medir la capacidad del niño ante una tarea de interferencia, más que para evaluar su conocimiento numérico.

\section{Referencias}

Brannon, E. M., Cantlon, J. F. y Terrace, H. S. (2006). The Role of Reference Points in Ordinal Numerical Comparisons by Rhesus Macaques (Macaca Mulatta). Journal of Experimental Psychology: Animal Behavior Processes, 32(2), 120-134. doi: 10.1037/0097-7403.32.2.120

Dehaene, D. (1997). The Number Sense. How the Mind Creates Mathematics. Oxford: Oxford University Press.

Donaldson, M. y McGarrigle, J. (1974). Some Clues to the Nature of Semantic Development. Journal of Child Language, 1(2), 185-194.

Frith, U. (2004). Autism: Explaining the Enigma. Oxford: Blackwell.

Gréco, P., Inhelder, B., Matalon, B. y Piaget, J. (1963). La formation des raisonnements recurrentiels. Paris: Presses Universitaires de France.

Karmiloff-Smith, A. (1996). Annotation: The Extraordinary Cognitive Journey from Foetus Through Infancy. Annual Progress in Child Psychiatry y Child Development, 1, 5-31.

McGarrigle, J. y Donalson, M. (1974). Conservation Accidents. Cognition, 3, 341-350.

Mehler, J. y Bever, T.G. (1967). Cognitive Capacity of very Young Children. Science, 158(3797), 141-142.

Navarro, J. I., Aguilar, M., Marchena, E., Ruiz, G., Menacho, I. y Van Luit, H. (2012). Longitudinal Study of Low and High Achievers in Early Mathematics. British Journal of Educational Psychology, 82, 28-41. doi: 10.1111/j.20448279.2011.02043.x

Ouden, H., Frith, U., Frith, C. y Blakemore, S. (2005). Thinking about Intentions. NeuroImage, 28, 787-796.

Piazza, M., Dehaene, S. y Gazzaniga, M. (2004). From Number Neurons to Mental Arithmetic: The Cognitive Neuroscience of Number Sense. En M. Piazza, S. Dehaene y M. Gazzaniga (Eds.). The Cognitive Neurosciences (pp. 865-875). Cambridge, MA: MIT Press.

Santrock, J. W. (2010). Life-span development. New York, NY: McGraw-Hill. 
Swanson, H. L., Jerman, O. y Zheng, X. (2008). Growth in Working Memory and Mathematical Problem Solving in Children at Risk and not at Risk for Serious Math Difficulties. Journal of Educational Psychology, 2, 343-379. doi: 10.1037/0022-0663.100.2.343

Thomas, M. y Karmiloff-Smith, A. (2003). Connectionist models of development, developmental disorders, and individual differences. En R. J. Sternberg, J. Lautrey y
T. I. Lubart (Eds.), Models of Intelligence: International Perspectives (pp. 133-150). Washington, D. C.: American Psychological Association. 